\title{
Síndrome de MünchHausen: diagnóstico e Manedo Clínico
}

\author{
Wagner F. Gattaz*, Harald Dressing, Walter Hewer, Paula Nunes \\ Trabalho realizado no Instituto Central de Saúde Mental, Mannheim, Alemanha e Departamento \\ e Instituto de Psiquiatria, Faculdade de Medicina, Universidade de São Paulo, SP.
}

RESUMO - Os autores apresentam uma revisão da literatura sobre a síndrome de Münchhausen, ilustrada pela descrição de um caso clínico bastante característico do transtôrno. Discute-se o diagnóstico e o diagnóstico diferencial com a simulação, a esquizofrenia e transtornos somatoformes tais como somatização e conversão. É importante que 0 clínico e 0 cirurgião estejam alertas para 0 diagnóstico de Münchhausen, porque esses pacientes se expõem a graves complicações de procedimentos clínicos e cirúrgicos desnecessários. Devido à baixa adesão ao tratamento, o manejo clínico da síndrome de Münchhausen é difícil. 0 diagnóstico precoce pode, de maneira considerável, excluir estes riscos iatrogênicos. Recomendamos que os pacientes com as características psicopatológicas da síndrome de Münchhausen sejam observados de forma cautelosa. Deve-se tentar clarificar tanto o diagnóstico clínico quanto psiquiátrico antes de se realizar qualquer procedimento invasivo.

UNITERMOS: Síndrome de Münchhausen. Transtorno factício. Transtorno somatoforme.

\section{INTRODUÇÃO}

Nota-Publicamos em 1990 uma primeira versão desta revisão em um periódico psiquiátrico (Gattaz WF, Dressing H e Hewer W, Münchhausen syndrome: Psychopathology and management, Psychopathology 23: 3339, 1990). Todavia, apenas uma minoria dos casos da síndrome de Münchhausen são atendidos por um psiquiatra. Pelas próprias características do quadro, a maioria destes pacientes perambula entre inúmeros hospitais, sendo atendidos nos diferentes serviços de clínica e de cirurgia de emergência. Assim, decidimos preparar esta versão atualizada do trabalho para divulgação no meio médico geral, no sentido de alertar e orientar a conduta diagnóstica e o encaminhamento terapêutico.

Em 1951, Asher cunhou o termo "Síndrome de Münchhausen" para descrever indivíduos que intencionalmente produziam e apresentavam sintomas físicos para receber tratamento hospitalar freqüente. Características associadas eram mentira patológica (pseudologia fantástica) e a passagem por diversos lugares para receber tratamento. Asher identificou três tipos: abdômen agudo, o mais fre-

\footnotetext{
*Correspondência:

Departamento de Psiquiatria da Faculdade de Medicina da Universidade de São Paulo R. Dr. Ovídio Pires de Campos s/n 05403-010 São Paulo - SP

E-mail: gattaz@usp.br - Fone: (+5511) 30629029 Fax: $(+5511) 30836588$
}

qüente, caracterizado por sintomas abdominais recorrentes e evidências de múltiplas laparotomias; hemorrágico, caracterizado por episódios de sangramento por múltiplos orifícios; neurológico, com ataques epilépticos, desmaios, dores de cabeça e anestesias.

Após o artigo de Asher, vários tipos foram adicionados na forma de relatos de caso (freqüentemente com a grafia incorreta "Munchausen") nos quais a sintomatologia artefatual afetava a maioria dos órgãos e sistemas $^{2}$. Sua caracterização com base em órgãos e sistemas envolvidos vem sendo, no entanto, criticada ${ }^{3}$, pois geralmente as queixas simuladas afetam simultaneamenteórgãos diferentes ou variam no curso da doença ${ }^{4}$.

Na quarta edição do Manual Diagnósticoe Estatístico das Doenças Mentais - DSM-IV5, a síndrome de Münchhausen está classificada no grupo dos transtornos factícios com o dígito 300.19 (transtorno factício com sintomas predominantementefísicos). Os critérios diagnósticos são: A) produção ou simulação intencional de sintomas e sinais predominantemente físicos; B) o papel de doente é o que motiva o comportamento; $C$ ) ausência de incentivos externos para o comportamento (ganho econômico, fuga de responsabilidade legal ou melhora de bem-estar físico). Não pode ocorrer exclusivamente durante o curso de uma outra doença do eixo I, como por exemplo a esquizofrenia.

Três outras categorias estão inclusas nos critérios do DSM-IV: transtorno factício com sintomas predominantemente psicológicos
(300.16), transtorno factício com sintomas psicológicos e físicos (300.19) e transtorno factício sem outra especificação (300.19). Fazer a diferenciação de transtorno factício meramente com base em sintomas físicos e psicológicos pode, no entanto, não ser adequado, uma vez que geralmente ambos estão presentes ${ }^{3}$.

$\mathrm{Na}$ Classificação de Transtornos Mentais e de Comportamento da CID-106 a síndrome de Münchhausen está classificada dentro dos transtornos factícios (F68.I).

Para ilustrar o quadro clínico, descreveremos a seguir um caso de uma paciente com quadro bastante característico de um transtorno factício com sintomas físicos e psicológicos. Apenas como curiosidade, durante sua "carreira" com a síndrome de Münchhausen, esta paciente causou um custo de aproximadamente 300 mil dólares ao seu seguro-saúde na Alemanha, além, naturalmente, dos riscos aos quais ela se expôs.

\section{Relato de Caso}

Uma mulher de 25 anos buscou, por conta própria, o nosso atendimento de emergência no Instituto Central de Saúde Mental de Mannheim, na Alemanha, relatando pensamentos suicidas. Uma investigação mais detaIhada revelou que sua queixa principal consistia de problemas físicos: sofria de dores no tórax que começaram agudamente e estavam conectadas com a respiração; além disso, caiu quatro vezes nas últimas horas antes de vir à 
sala de observação. Essas descrições exatas e concisas das queixas pareciam plausíveis e sugeriam que esta paciente extremamente obesa (I68 cm, I I 4 kg) sofria de embolismo pulmonar.

No entanto, os dados subseqüentes da história pareciam muito vagos e levantaram suspeita. Dezoito dias antes tinha sido laparotomizada em Hamburgo devido a gravidez extrauterina. Sete dias depois, saiu do hospital mesmo contra as orientações médicas. Viajou (aproximadamente $700 \mathrm{~km}$ ) para Stuttgart e referiu ter desmaido. Foi levada a um pronto-socorro e lá encontrava-se em estado de turvação de consciência. Novamente laparotomizada, uma flebotrombose foi removida de sua pelve. Mais uma vez fugiu, e três dias depois, em outro hospital, foi submetida a uma cirurgia por causa de um abscesso na parede abdominal. Muito embora estivesse com drenos e cuidado cirúrgico complementar fosse necessário, saiu do hospital por conta própria.

A paciente observou que não podia entender seu comportamento nestas últimas semanas. Por algum motivo, quando sentia uma sensação de pânico aumentar, não conseguia resistir ao impulso de sair correndo. Ela sabia estar arriscando muito a sua vida e por causa disso decidiu procurar um hospital psiquiátrico para poder finalmente ser ajudada.

O exame físico revelou uma enorme lesão supurada no abdômen inferior englobando os três drenos e chegando até a fáscia. Não havia evidência de sintomatologia psicoorgânica ou psicótica. Uma das características marcantes eram os dados vagos, imprecisos e parcialmente inconsistentes sobre sua história. Ela parecia bastante amigável, mas afetivamente indiferente e displicente. Seu comportamento social era ligeiramente inadequado. Apoiava suas colocações com o uso correto de terminologia médica (dizia ser enfermeira, mas posteriormente descobriu-se que esse dado estava incorreto). Seus comentários, depreciativos em relação ao cuidado médico durante as hospitalizações passadas bem como suas descrições extremamente positivas de nosso hospital, chamavam a atenção.

Apesar das condições um tanto quanto estranhas no primeiro contato (cuja importância poderia ser entendida muito mais tarde) a paciente foi imediatamente transferida para o departamento clínico. Objetivava-se avaliar melhor as queixas somáticas, fazer uma cintilografia pulmonar e cirurgia complementar para a lesão abdominal. Através da cintilografia, um êmbolo pulmonar não pôde ser descartado. A abordagem da lesão abdominal requeria pelo menos algumas semanas de hospitalização. Isso parecia exposição a mais risco, dada a história de múltiplas fugas do hospital. Desta forma, solicitamos uma autorização sua para ser admitida em nossa unidade de internação fechada.

Ela forneceu mais detalhes de sua história (os quais, no entanto, permaneceram fragmentados e parcialmente mentirosos) e nos autorizou investigar com sua seguradora de saúde dados a respeito de suas hospitalizações prévias. Descobrimos que nos últimos três meses tinha sido internada em mais de 20 hospitais. Esteve inúmeras vezes em setores de clínica médica, ginecologia, urologia, cirurgia e psiquiatria em toda Alemanha alegando: dores agudas no abdômen (dizia não ter menstruado e que o teste de gravidez foi positivo), sintomas de um êmbolo pulmonar, desmaio, febre, hipertonia (um feocromocitoma havia sido descoberto), estados de irritabilidade e ideação suicida.

O curso das suas internações - das quais repetidamente escapou após um curto período-exames e procedimentos cirúrgicos foram com freqüência arriscados e perigosos. Por exemplo, teve pelo menos seis laparotomias de emergência (e provavelmente até mais) e também abrasões, várias laparoscopias, colposcopias e citoscopias. Diversas vezes saiu do hospital imediatamente após a operação, chegando a sair com um cateter subclávio e drenos. Vários raios- $X$, cintilogramas pulmonares e flebografias foram feitos. Tomou drogas de alta potência como morfina, anticoagulantes, antibióticos e antihipertensivos.

Além das questões médicas, a paciente despertou considerável atenção devido ao seu transtorno de personalidade. Nos últimos anos passou trotes para a polícia, bombeirose ambulância dizendo, por exemplo, que havia tomado uma dose letal de droga. Recebeu diversas advertências por abusar de chamadas de emergência. Somado a isso, repetidas vezes roubou dinheiro e jóias de familiares, amigos e estranhos. Conheceu homens e ganhou confiança e ajuda ao fingir estar desamparada. Abusou dessa confiança e roubou grandes somas em dinheiro, cheques e cadernetas de poupança deixando estas pessoas com dívidas.

Com intensidade variável, a sintomatologia podia ser reconhecida há pelo menos sete anos, quando teve um tratamento psiquiátrico. Tentou suicídio com medicamentos e recebeu o diagnóstico de personalidade infantil histérica com desenvolvimento antisocial. Naquela época, já simulava estupros, gravidez e abortos e teve diversas admissões forçadas em hospitais.

Para obter uma história objetiva, contatamos à mãe da paciente. A paciente tinha um irmão cinco anos mais novo e uma irmã quatro anos mais nova, ambos sem história psiquiátrica. Quando tinha II anos, seus pais se separaram (o pai era um arquiteto autônomo, a mãe dona-de-casa). Os filhos do casal ficaram, por algum tempo, com uma família tutelar e depois foram criados pela mãe. Desde criança já era notório o quanto escapava e faltava à escola, mentia e fraudava.

Quando terminou a escola, estava sob custódia de uma moradia terapêutica e pedagógica, pois a família não conseguia mais controlá-la. Conseguiu concluir um treinamento em economia doméstica. Resolveu exercer a função por um ano, mas logo foi colocada sob aviso, uma vez que freqüentemente faltava sem justificativa. Passou a mudar sempre de lugar, tinha relacionamentos superficiais e passageiros, viveu em hotéis sem pagar as contas ou em admissões forçadas em hospitais com falsas pretensões.

Em 1982, foi presa e avaliada por um perito em psiquiatria. Asentença de três anos de prisão foi suspensa e em seu lugar precisou cumprir tratamento psicoterápico sob custódia. Sem sucesso, a terapia precisou ser interrompida, pois tinha tendência a atuar. Como conseqüência, foi presa por aproximadamente meio ano. Depois disso, foi liberada sob custódia. De 1985 a 1988 teve um relacionamento firme e razoavelmente estável com um homem e com ele teve um filho. Era, no entanto, negligente com a criança, não a alimentava ou trocava as fraldas, voltou a contrair dívidas, tinha longas conversas com a polícia e bombeiros e deflagrou várias ações de emergência ao dizer, por exemplo, que tomou medicamentos ou que seu bebê foi morto. Após a separação, iniciou a mencionada peregrinação por hospitais.

Por meio de cuidado psicoterapêutico intensivo, tentamos nos aproximar da paciente e 
Tabela I - Características associadas à síndrome de Münchhausen

História extensa deviagem (errante) pelo país buscando umnovo hospital para ser internado

Hospitalizações curtas, interrompidas por saídas contra orientaçãomédica

História dramática com um conhecimento extenso de terminologia médica sem, no entanto, participação emocional (belleindiférence)

Inconsistênciasnahistória

Pseudologiafantástica

Sintomatologiaflutuante

"Abdômenem grelha" devido aos procedimentos cirúrgicos múltiplos

Históriacriminal

por aproximadamente seis semanas conseguimos mantê-la em contato próximo. No entanto, o trabalho psicoterapêutico foi sempre dificultado por suas extensas pseudologias. Por exemplo, fez declarações falsas sobre o seu curriculum vitae, dizia ter completado o segundo grau e recebido treinamento como enfermeira. Alegava ter sido estuprada diversas vezes; dizia ter sido molestada sexualmente pela primeira vez por seu pai quando tinha 10 anos. Sem aviso prévio e de forma surpreendente, um dia a paciente não retornou de um passeio externo que lhe havia sido permitido.

Pelo contato com hospitais que seguiram o tratamento da paciente, descobrimos que um dia após sair do nosso serviço ela simulou sintomas abdominais e foi novamente laparotomizada. Alguns dias depois, o mesmo procedimento cirúrgico foi realizado em um terceiro hospital. Posteriormente, foi internada por um curto período em um hospital psiquiátrico devido a comportamentos suicidas. Não dispomos de mais informações sobre esta paciente, mas provavelmente mais intervenções cirúrgicas e procedimentos diagnósticos foram realizados.

\section{Discussão}

Nossa paciente preenchia os critérios do DSM-IV para transtorno factício com sintomas físicos e psíquicos. Foi internada diversas vezes devido a queixas somáticas e foi admitida duas vezes em hospitais psiquiátricos devido a depressão e pensamentos suicidas. Mais ainda, apresentava em sua anamnese as diferentes características resumidas na Tabela I, que são características da síndrome de Münchhausen tal qual Asher definia ${ }^{7}$ : Deve-se suspeitar da síndrome de Münchhausen em pacientes apresentando essas características. Outros sinais que reforçam a suspeita são a não resposta ao tratamento ou resposta placebo e exacerbação ou produção de sintomas quando o paciente está sendo ciente de ser observada ${ }^{8}$. A prevalência dos transtornos factícios é desconhecida. Provavelmente, é bem mais comum do que se diagnostica. Com freqüência acomete profissionais da área de enfermagem e em geral se manifesta entre os 20 e 30 anos. Na síndrome de Müchhausen encontrase uma maior prevalência de homens ${ }^{9}$. Desta forma, para o diagnóstico, são necessários uma anamnese detalhada e um exame físico cauteloso. São essenciais também dados vindos da família (sempre que possível) e de outros relatos médicos.

O diagnóstico diferencial mais importante é, obviamente, uma doença física verdadeira. Estes pacientes têm um risco aumentado para doenças físicas como conseqüência de seu comportamento autodestrutivo ou suas complicações iatrogênicas induzidas (por exemplo, por tratamentos cirúrgicos e clínicos prévios). Enoch e Trethowan ${ }^{2}$ citaram um caso em que o paciente morreu porque a equipe médica recusou-se a realizar uma cirurgia para abdômen agudo. Síndromes Mentais Orgânicas, como por exemplo as lesões fronto-parietais, principalmente no hemisfério direito, podem, da mesma forma, apresentar os mesmos sintomas psíquicos ${ }^{10,11,12}$.

O diagnóstico diferencial de transtornos somatoformes, tais como transtorno conversivo e transtorno de somatização devem ser considerados. Tanto na síndrome de Münchhausen quanto nos transtornos somatoformes há a presença de sintomas sem a doença de base. Muito embora em ambas patologias a motivação seja inconsciente (a formação de sintomas é uma solução para um conflito psicológico), na síndrome de Münchhausen a produção de sintomas é intencional, pois a motivação primária está em se assumir o papel de doente. Esta diferenciação pode ser bem difícil ${ }^{13}$. Já a produção intencional de sintomas para se obter um ganho pessoal (motivação consciente), caracteriza a simulação e a diferencia dessas duas condições. Nesta última, o ganho secundário vem de recompensas externas, tais como benefícios financeiros, obtenção de aposentadoria ou escape de uma punição.

Para completar o diagnóstico diferencial, deve-se mencionar a esquizofrenia com sintomas cenestésicos. Esta é uma forma de psicose com uma predominância de alucinações corporais que muitas vezes se apresenta com queixas físicas ${ }^{14}$. No entanto, a ausência de sintomas psicóticos na síndrome de Münchhausen geralmente permite o diagnóstico diferencial sem maiores dificuldades.

A síndrome de Münchhausen está freqüentemente associada a um transtorno de personalidade, outro diagnóstico do eixo II (DSM-IV). Na nossa paciente, um transtorno de personalidade borderlinegrave foi diagnosticado com base nos seguintes critérios: I) comportamento impulsivo, imprevisível tal como seus roubos, ingestão descontrolada com adiposidade maciça e atos autodestrutivos ao provocar procedimentos cirúrgicos arriscados e desnecessários; 2) relacionamentos interpessoais intensos mas instáveis que oscilavam entre idealização e depreciação, particularmente aparente na relação terapêutica mas também no tipo e duração de parcerias descritas acima; 3 ) instabilidade afetiva: durante o período de observação dentro do hospital alternava fases de raiva inapropriadae irritabilidade com amizade, ajustamento e solicitude; 4) insegurança óbvia de identidade com autopercepção controversa e vaga; idéias irrealistas sobre o futuro; percepção dos outros superficial, rasa e limitada; 5) dificilmente tolerava estar sozinha: durante o período de observação continuamente buscava contato e afeição; a história de longas e freqüentes conversas ao telefone com a polícia, bombeiros, etc também devem ser mencionadas; (6) autodestrutividade física devido ao grande número de procedimentos cirúrgicos induzidos por pretensões falsas.

Como com a nossa paciente, o transtorno de personalidade borderline é provavel- 
mente o diagnóstico mais freqüentemente associadoà síndrome de Münchhausen $n^{4,15,16,17,18}$. A coexistência freqüente de características anti-sociais não justifica, neste caso, o diagnóstico de transtorno de personalidade anti-social, uma vez que este último não está associado ao comportamento que busca hospitalizações visto na síndrome de Münchhausen.

O principal objetivo do tratamento da síndrome de Münchhausen é evitar dano extra ao se prevenir procedimentos médicos desnecessários. O segundo objetivo é conseguir motivar o paciente para uma relação terapêutica ${ }^{9}$. Até o momento, as abordagens terapêuticas mostraram resultados insatisfatórios e não existe consenso quanto ao meIhor tratamento ${ }^{13,19}$. Cada internação pode reforçar os temores dos pacientes de que pode existir algo de grave que justifique os procedimentos diagnósticos invasivo ${ }^{20}$. Apesar da necessidade óbvia por tratamento psiquiátrico, apenas uma porcentagem muito pequena é encaminhada para o psiquiatra ${ }^{21}$. Isto é provavelmente conseqüenteà tendência humana de negar a existência de uma doença (inclusive psiquiátrica) quando a simulação é descoberta e uma doença física é descartada. $O$ zelo inicial da equipe do hospital é substituído por uma sensação de fracasso e conseqüentes sentimentos de frustração, raiva $\mathrm{e}$ eventualmente rejeição ${ }^{22}$. Ao invés de ser encaminhado para o tratamento psiquiátrico, os pacientes podem ser rotulados de mentirosos ou simuladores e em alguns casos são submetidos a processos legais ou de punição $0^{23}$.

Quando confrontados com sua história, esses pacientes geralmente partem contra as orientações médicas para recomeçar a busca por outra admissão hospitalar. Kellner e Eth ${ }^{24}$ sugeriram que evitar o confronto com estes pacientes pode levar a um resultado terapêutico melhor. Deixar o paciente saber paulatinamente sobre sua condição traz mais chances de cooperação $0^{25}$. $O$ paciente tem mais chances de aceitar ajuda psiquiátrica se sentir que o seu comportamento é aceito e entendido como uma expressão de fatores psicológicos (isto é, inconscientes). Uma vez que o paciente deseja saber que não está medicamente doente e deseja se redefinir como doente psiquiátrico, a psicoterapia pode ser possível ${ }^{26}$.

Não existe até o momento uma aborda- gem psicoterapêutica que se provou útil no manejo da síndrome de Münchhausen. Cremona-Barbaro ${ }^{27}$ sugeriu uma análise psicodinâmica profunda, mas não forneceu nenhuma estratégia terapêutica. Enoche Trethowan ${ }^{2}$ discutiram a impossibilidade prática dos métodos psicoanalíticos nestes casos, mas salientaram a necessidade de psicoterapia de suporte prolongada "pelo menos para manter estes pacientes fora dos departamentos clínicos e cirúrgicos dos hospitais". Powell e Boast ${ }^{19}$ sugerem técnicas cognitivo-comportamentais para lidar com problemas, treino de habilidades sociais e psicoeducação. O objetivo seria promover reintegração social e reduzir o comportamento inadequado. O grande problema é que tais técnicas exigem a cooperação do paciente ${ }^{23}$.

Tanto antidepressivos quanto neurolépticos já foram utilizados para o tratamento. Como é de se imaginar, antidepressivos são, em geral, benéficos quando há um transtorno afetivo concomitante?.

Como já mencionado, é evidente que pacientes com a síndrome de Münchhausen se expõem a altos riscos os quais são, de maneira considerável, induzidos de forma iatrogênica. Protegê-los destes riscos é uma tarefa de máxima relevância. Janofsky ${ }^{28}$ sugere que deve ser obrigatório para pacientes com uma história médica complexa e várias internações prévias fornecer um registro de todas as internações prévias antes de sua admissão eletiva no hospital. Apesar de seu valor teórico, consideramos que esta medida não tem aplicações práticas para a maioria dos pacientes diante de circunstâncias normais e ainda menos em uma situação de emergência. Markantonakis e Lee ${ }^{29}$ propuseram o estabelecimento de um registro central que poderia conter descrições do paciente, os nomes utilizados, padrões anteriores de apresentação e fornecer informação telefônica quando necessário. Acreditamos que o estabelecimento deste registro pode fornecer uma ajuda definitiva na identificação precoce destes pacientes e desta forma evitar ao menos mutilações induzidas iatrogenicamente. No entanto, ao menos na Alemanha, este projeto não tem apoio da legislação vigente de proteção de informações pessoais ${ }^{30}$.

Como na maioria das áreas médicas, o diagnóstico precoce da síndrome de Münchhausen é o meio mais efetivo de tratar adequadamente estes pacientes, ou pelo menos evitar complicações induzidas iatrogenicamente. Recomendamos, desta forma, que pacientes que apresentam as características da síndrome de Münchhausen (Tabela I) devem ser observados de forma cuidadosa e conservadora o maior período de tempo possível (se necessário em terapia intensiva) e um esforço para esclarecer o diagnóstico deve ser feito antes de se realizar qualquer procedimento invasivo.

\section{SUMMARY}

MÜNCHHAUSEN SYNDROME: DIAGNOSIS AND MANAGEMENT

The authors review the literature on Münchhausen syndrome, and ilustrate the clinical features of the disorder with the description of a characteristic case. Diagnosis and differential diagnosis are discussed with regard to other somatoform disorders such as conversiondisorderand somatizationdisorderas well as to malingering and schizophrenia. The awareness of general practitioners and surgeons regarding thissyndromemayavoid the exposure of these patients to serious complications of unnecessary medical and surgical procedures. The management of Müchhausen syndrome is aggravated by the low compliance in these patients. Early diagnosis could to a considerable extent prevent the iatrogenic risks. The authors recommend that patients presenting the psychopathological features of a Münchhausen syndrome should be conservatively observed and an attempt to clarify both the medical and the psychiatric diagnosisshould be made beforeany invasive procedure is undertaken. [RevAssoc Med Bras 2003; 49(2): 220-4]

KEY WORDS: Münchhausen syndrome. Factitious disorder. Somatoform disorders.

\section{REFERÊNCIAS}

I. Ody R. Münchhausens's syndrome? Br J Psychiatry 1993; 163:416-7.

2. Enoch MD, Trethowan WH. The Munchausen syndrome and some related disorders. In: Uncomon psychiatric syndromes. Bristol: Wright; 1979. p.77-94.

3. Sussman N, Borod JC, Cancelmo JA, Braun D. Munchausen's syndrome: areconceptualization of the disorder. J Nerv Ment Dis 1987; I75:692-5.

4. Bock KD, Overkamp F. Vorgetäuschte Krankeheit. Klin Wochenchr 1986; 64: 149-64.

5. American Psychiatric Association (APA). Diagnostic and statistical manual of mental 
disorders. 4th ed. Washington: American Psychiatric Association; 1994. p.47I-5.

6. World Health Organization. The ICD-IO Classification of Mental and Behavioural Disorders. Geneva: World Health Organization; 1992.

7. Asher R. Muchausen's syndrome. Lancet I951; I:339-41.

8. Popli AP, Masand PS, Dewan MJ. Factitious Disorders with Psychological Symptoms.J Clin Psychiatry 1992; 53:315-8.

9. Wiley SD. Deception and detection in psychiatric diagnosis. Psychiatr Clin North Am 1998; 21:869-93.

10. Fenelon G, Mahieux F, Roullet E, Guillard A. Muchausen's Syndrome and abnormalities on magnetic resonance imaging of the brain. BMJ 1991; 302:996-7.

II. Lawrie SM, Goodwin G, Masterton G. Muchausen's Syndrome and Organic Brain Disorder. Br JPsychiatry 1993; 162:545-9.

12. Hedges BE, DimsdaleJE, Hoyt DB. Muchausen Syndrome presenting as recurret multiple trauma. Psychosomatics 1995; 36:60-3.

13. Feldman MD, Duval NJ. Factitious quadriplegia: a rare new case and literature review. Psychosomatics 1997; 38:76-80.
14. Huber G. Pneumoencephalographische und psychopathologische Bilder bei endogenen Psychosen. Berlin: Springer; 1957.

15. Nadelson T. The Muchausen spectrum: borderline character features. Gen Hosp Psychiatry 1979; I: I I-7

16. Ries RK. DSM-III differential diagnosis of Muchausen's syndrome. J Nerv Ment Dis 1980; 168:629-32.

17. Ford CV. The somatizing disorders. Amsterdam: Elsevier; 1983.

18. Baile WF, Kuehn CV, Straker D. Factitious cancer. Psychosomatics 1992; 33: 100-5.

19. Powell R, Boast N. The million dollar man: resource implications for chronic Munchausen's Syndrome. Br J Psychiatry 1993; 162:253-6.

20. Higgins PM. Temporary Munchausen Syndrome. Br J Psychiatry 1990; I57:6 I 3-6.

21. Spiro HR. Chronic factitious illness. Arch Gen Psychiatry 1968; 18:569-79.

22. London M, Ghaffari K. Munchausen's syndrome and drug dependence. Br J Psychiatry 1986; |49:65 I-4.

23. Black W. Resource implications of Muchausen's Syndrome. Br J Psychiatry 1993; 162:847-8.
24. Kellner $\mathrm{CH}$, Eth $\mathrm{S}$. Code blue-factitious cyanosis. J Nerv Ment Dis 1982; I 70:37I-2.

25. Lyell A. Dermatitis artefacta and self-inflicted disease. Scott Med J 1972; 17:187-96.

26. Hollender MH, Hersh SP. Impossible consultation made possible. Arch Gen Psychiatry 1970; 23:343-5.

27. Cremona-Barbaro A. The Muchausen syndrome and its symbolic significance: an in-depth case analysis. Br J Psychiaty 1987; I 5 I:76-9.

28. JanofskyJS. Muchausen syndrome in a mother and daughter: an unusual presentation of folie à deux. J Nerv Ment Dis 1986; 174:368-70.

29. Markantonakis A, Lee AS. Psychiatric Munchausen's syndrome: a college register ? (letter). Br J Psychiatry 1988; I 52:867.

30. Rieger HJ. Allgemeines Persönlichkeitsrecht und Schweigepflicht ber der Behandlung von Simulanten. Dtsch Med Wochenchr 1975; 100:567-68.

Artigo recebido: 07/08/02 Aceito para publicação: 29/08/02 